\title{
Sulforaphane protects against oxidative stress-induced apoptosis via activating SIRT1 in mouse osteoarthritis
}

\author{
MANGMANG CHEN ${ }^{1,2}$, LIPENG HUANG ${ }^{2}$, YANGXUN LV ${ }^{2}$, LIUBING LI $^{1}$ and QIRONG DONG ${ }^{1}$ \\ ${ }^{1}$ Department of Orthopedics, The Second Affiliated Hospital of Soochow University, Suzhou, Jiangsu 215004; \\ ${ }^{2}$ Department of Orthopedics Surgery, The Dingli Clinical Institute of Wenzhou Medical University, \\ Wenzhou Central Hospital, Wenzhou, Zhejiang 325000, P.R. China
}

Received August 8, 2020; Accepted February 26, 2021

DOI: $10.3892 / \mathrm{mmr} .2021 .12251$

\begin{abstract}
Osteoarthritis (OA), the most common form of human joint disease, is characterized by progressive degeneration of the articular cartilage, synovitis and subchondral osteoporosis. Chondrocyte apoptosis is the primary pathogenic mechanism of $\mathrm{OA}$ and is considered to be a potential therapeutic target. Sulforaphane (SFN), a dietary isothiocyanate obtained from cruciferous vegetables, has been reported to exert an anti-apoptotic effect by activating sirtuin 1 (SIRT1). To the best of our knowledge, however, the effects of SFN on apoptotic responses in OA have not been reported. In the present study, SFN was shown to significantly inhibit chondrocyte apoptosis while enhancing expression levels of SIRT1 in a $\mathrm{H}_{2} \mathrm{O}_{2}$-induced $\mathrm{OA}$ mouse model. The anti-apoptotic effect of SFN was reversed by SIRT1 small interfering RNA, implying that SIRT1 exerted a protective role against the effect of SFN on chondrocytes. The expression levels of C/EBP homologous protein, 78-kDa glucose regulated protein, Bax, Bcl-2 and cleaved caspase 3 were found to be downregulated in SFN-treated mice. Furthermore, SFN ameliorated cartilage degradation in the OA mouse model. These findings indicate that SFN exerted an anti-apoptotic effect on chondrocytes and ameliorated OA in vivo by activating the SIRT1 signaling pathway.
\end{abstract}

\section{Introduction}

Osteoarthritis (OA), a degenerative disease of joints, is the most common cause of musculoskeletal disability $(1,2)$. Various factors, such as age, genetics, adiposis and sex, are involved

Correspondence to: Dr Qirong Dong or Dr Liubing Li, Department of Orthopedics, The Second Affiliated Hospital of Soochow University, 1055 San Xiang Road, Suzhou, Jiangsu 215004, P.R. China

E-mail: dongqirong@suda.edu.cn

E-mail:1bli@suda.edu.cn

Key words: osteoarthritis, sulforaphane, chondrocyte, inflammation, sirtuin 1 in the development of osteoarthritis (3). Destroyed cartilage structure and loss of function are the central hallmarks of OA (4). Although studies have revealed potential mechanisms involved in the progression of OA, its pathological etiology is unknown. Moreover, there are no effective therapeutic options that can prevent disease progression $(5,6)$. OA is associated with degradation of matrix cartilage and chondrocytes, which are the only type of cell in articular cartilage (7). Extracellular matrix (ECM) molecules are involved in maintaining the structure and function of regular cartilage, whose components include proteoglycans and collagen, produced by chondrocytes (8). Articular cartilage degradation is initiated by several inflammatory cytokines (IL-6, TNF- $\alpha$, prostaglandin 2) and reactive oxygen species (ROS) that induce oxidative stress (9). Excessive chondrocyte apoptosis and senescence occur due to ROS accumulation (10). Therefore, inhibiting chondrocyte apoptosis and senescence is a potential strategy for preventing articular cartilage degradation in OA.

Endoplasmic reticulum (ER), which is the first site in the secretory pathway, synthesize and fold proteins (11). ER regulate the balance between pathological and physiological conditions (12-14). The ER stress (ERS) response enhances cell survival by decreasing the load of unfolded proteins. However, cells undergo apoptosis when ERS is overwhelming (15). ERS-associated apoptosis is an important signaling pathway during apoptosis (16). It has been demonstrated that ERS signaling in this pathway promotes repair of the unfolded protein response (UPR) of the ER (17). UPR serves a critical role in cell growth regulation, differentiation and apoptosis (18). ERS has been shown to stimulate the $\mathrm{C} / \mathrm{EBP}-$ homologous protein $(\mathrm{CHOP})$ pathway while activating glucose-regulated protein 78 (GRP78) (19). The Bcl-2 protein family acts against apoptosis by inhibiting apoptotic mediators, such as Bax and Cytochrome c (20). Studies have reported that ER and mitochondrial pathways regulate apoptosis during rat intervertebral disc degeneration $(21,22)$. These findings show the importance of ER and mitochondria in chondrocytes.

There are seven known members of mammalian Sirtuins (SIRTs). Among them, SIRT1 regulates multiple functions in metabolic syndrome, oxidative stress, inflammation and aging (23-25). Li et al (26) reported that sulforaphane (SFN) can downregulate ER-induced apoptosis protein expression levels in a rat model of hypoxia/reoxygenation injury 
by activating SIRT1. Activated SIRT1 inhibits oxidative damage and inflammation (26) and is therefore a therapeutic target for OA via regulating mitochondrial biogenesis (27). Feng et al (28) reported that curcumin exerts a protective effect against OA by activating SIRT1.

SFN (a type of dietary isothiocyanate) is primarily found in cruciferous vegetables (29). It exhibits various pharmacological properties, including anti-cancer, anti-inflammatory and anti-oxidative stress (30). SFN exerts a protective effect during post-ischemic cardiac injury (31). Moreover, it exerts cardioprotective effects by activating SIRT1 and suppressing ER stress (32). Davidson et al (33) reported that SFN inhibits inflammation via the $N F-\kappa B$ signaling pathway in $\mathrm{OA}$ mice. Moreover, they found that SFN suppresses IL-1/NF- $\kappa$ B and Wnt3a/T cell factor/lymphoid enhancer factor signaling while enhancing TGF $\beta / \mathrm{SMAD} 2 / 3$ and bone morphogenetic protein 6/SMAD1/5/8 signaling in IL-1/oncostatin M-induced chondrocytes (34). However, its anti-apoptotic effects during OA have not been established. The aim of the present study was to elucidate the role of SFN in apoptosis, including its specific mechanisms of action, in $\mathrm{H}_{2} \mathrm{O}_{2}$-treated chondrocytes. The effect of SFN on cartilage degeneration was investigated in a mouse model of OA.

\section{Materials and methods}

Reagents and antibodies. SFN (purity $\geq 98 \%$ ), Safranin O and toluidine blue stain were obtained from Beyotime Institute of Biotechnology; collagenase type II, $\mathrm{H}_{2} \mathrm{O}_{2}$ and DMSO were bought from Sigma-Aldrich (Merck KGaA). BCA protein assay kit was obtained from Beyotime Institute of Biotechnology; primary antibodies against GRP78, CHOP and GAPDH were obtained from Wuhan Sanying Biotechnology; primary antibodies against Bax, Bcl-2, Cleaved caspase-3 and SIRT1 were purchased from Cell Signaling Technology, Inc.; In Situ Cell Death Detection kit was obtained from Roche Diagnostics; secondary antibodies (cat. no. SA00001-14; Wuhan Sanying Biotechnology), including Goat Anti-Rabbit IgG (cat. no. B900210; Wuhan Sanying Biotechnology) and Alexa Fluor ${ }^{\circledR}$ 488-labeled goat anti-rabbit IgG $(\mathrm{H}+\mathrm{L})$ secondary antibody (cat. no. SA00009-3; Wuhan Sanying Biotechnology), were obtained from Jackson ImmunoResearch Laboratories, Inc.; Cell-Counting Kit-8 (CCK-8) was purchased from Dojindo Molecular Technologies, Inc. DMEM-F12 was purchased from Gibco (Thermo Fisher Scientific, Inc.).

Primary chondrocyte isolation and culture. A total of 10 C57BL/6 mice (5 males and 5 females; age, 10 days; weight, $4 \mathrm{~g}$; the Animal Center of the Chinese Academy of Sciences) were euthanized by $100 \mathrm{mg} / \mathrm{kg}$ sodium pentobarbital. All animal procedures were performed in accordance with the Guidelines for Care and Use of Laboratory Animals of Wenzhou Medical University (35) and experiments were approved by the Animal Ethics Committee of Wenzhou Medical University. The knee cartilage of mice were collected carefully under aseptic conditions using a dissecting microscope, and tissue was treated with $2 \mathrm{mg} / \mathrm{ml}(0.1 \%)$ collagenase II for $4 \mathrm{~h}$ at $37^{\circ} \mathrm{C}$. The digested tissue was centrifuged $(800 \mathrm{x} \mathrm{g})$ for $5 \mathrm{~min}$ at $37^{\circ} \mathrm{C}$. Then, chondrocytes $\left(5 \times 10^{4}\right.$ cells $\left./ \mathrm{cm}^{2}\right)$ were seeded into culture flasks. Cells were cultured in DMEM/F12 supplemented with $10 \%$ fetal bovine serum and $1 \%$ antibiotics (penicillin/streptomycin) and incubated in an atmosphere of $5 \% \mathrm{CO}_{2}$ at $37^{\circ} \mathrm{C}$. The culture medium was replaced every 2-3 days. When 80-90\% confluency was attained, cells were fused and $0.25 \%$ trypsin-EDTA solution was used to subculture the chondrocytes. In order to avoid phenotypic loss, chondrocytes from the first and second channels were used.

Cell viability assay. CCK-8 assay was used to evaluate chondrocyte viability according to the manufacturer's instructions. First, chondrocytes were incubated in 96-well plates $(8,000$ cells/well $)$ for $24 \mathrm{~h}$. Then, they were treated with a concentration gradient $(0.0,12.5,25.0,50.0,100.0$ and $200.0 \mu \mathrm{M}$ ) of SFN for $24 \mathrm{~h}$ and $48 \mathrm{~h}$. Finally, $10 \mathrm{~mol} / \mathrm{l}$ CCK-8 solution was added to each well for $2 \mathrm{~h}$ at $37^{\circ} \mathrm{C}$, after which optical density was spectrophotometrically measured at $450 \mathrm{~nm}$ (Thermo Fisherr Scientific, Inc.).

Intracellular ROS production assay. Intracellular ROS levels were detected using the HDCFDA Probe Assay kit (Beyotime Institute of Biotechnology), according to the manufacturer's instructions. Intracellular ROS generation was measured at $485 \mathrm{~nm}$ (excitation) and $535 \mathrm{~nm}$ (emission) using a microplate reader (Bio-Rad Laboratories, Inc.).

Western blot analysis. In order to obtain total proteins from chondrocytes, RIPA lysis buffer (Beyotime Institute of Biotechnology) was added to $1 \mathrm{mM}$ PMSF and kept on ice for $10 \mathrm{~min}$, after which the mixture was centrifuged at $1,000 \mathrm{xg}$ for $15 \mathrm{~min}$ at $4^{\circ} \mathrm{C}$. BCA protein detection kit was used to estimate protein concentration. Then, $40 \mathrm{ng}$ protein was separated by SDS-PAGE (10\%) and transferred to polyvinylidene fluoride membrane. Membranes were blocked using $5 \%$ skimmed milk for $2 \mathrm{~h}$ at $37^{\circ} \mathrm{C}$ and detected overnight at $4^{\circ} \mathrm{C}$ with the following antibodies: CHOP $(1: 1,000$; cat. no. 15204-1-AP; Wuhan Sanying Biotechnology), GRP78 (1:1,000; cat. no. 11587-1-AP; Wuhan Sanying Biotechnology), Bcl-2 (1:1,000; cat. no. 15071; Cell Signaling Technology, Inc.), Bax (1:1,000; cat. no. 5023; Cell Signaling Technology, Inc.), Cleaved caspase-3 (1:1,000; cat. no. 9661; Cell Signaling Technology, Inc.), SIRT1 (1:500; cat. no. 2493; Cell Signaling Technology, Inc.) and GAPDH (1:1,000; cat. no. 10494-1-AP; Wuhan Sanying Biotechnology).

The membrane was washed using TBS-Tween-20 (TBST; 5\% Tween-20) and incubated with the corresponding secondary antibodies (1:1,000; cat. no. SA00001-14; Wuhan Sanying Biotechnology) for $2 \mathrm{~h}$ at room temperature. After being washed three times using TBST, the blots were visualized using electrochemiluminescence plus reagent (Invitrogen; Thermo Fisherr Scientific, Inc.). Image Lab 3.0 software (Bio-Rad Laboratories, Inc.) was used to measure the intensity of each band.

TUNEL assay. Apoptotic chondrocytes were measured by TUNEL staining using an In Situ Cell Death Detection kit, according to the manufacturer's instructions. The chondrocytes $\left(1 \times 10^{5}\right)$ were seeded in a 6 -well plate and treated with SFN $(50 \mu \mathrm{M})$ or $\mathrm{H}_{2} \mathrm{O}_{2}(50 \mu \mathrm{M})$ for $24 \mathrm{~h}$ at $37^{\circ} \mathrm{C}$, fixed in $4 \%$ paraformaldehyde for $45 \mathrm{~min}$ at $37^{\circ} \mathrm{C}$, incubated with $0.5 \%$ Triton $\mathrm{X}-100$ for $15 \mathrm{~min}$ at $37^{\circ} \mathrm{C}$ and washed using PBS 
for 5 min. Finally, cells were stained using the In Situ Cell Death Detection kit for $60 \mathrm{~min}$ at $65^{\circ} \mathrm{C}$, after which the nuclei were stained with DAPI for $1 \mathrm{~min}$ at $37^{\circ} \mathrm{C}$ (Beijing Solarbio Science \& Technology Co., Ltd.). In total, 25 fields of each slide were randomly selected and images were observed using a fluorescence microscope (magnification, x100; scale bar, $50 \mu \mathrm{m}$; Olympus Corporation).

Small interfering(si)RNAtransfectionfor 24h.Double-stranded siRNA for mouse SIRT1 gene silencing was purchased from Abcam. The SIRT1 siRNA sequence was as follows: 5'-UUG GGAUUCACGCAUAGGAGCACUG-3'. Cells $\left(1 \times 10^{5}\right)$ were seeded into a 6 -well plate, incubated for $24 \mathrm{~h}$ at $37^{\circ} \mathrm{C}$ and transfected with negative control [non-specific non-targeting siRNA (scramble); Abcam] or SIRT1 siRNA duplexes for $36 \mathrm{~h}$ at $50 \mathrm{nM}$ using Lipofectamine ${ }^{\circledR} 2000$ siRNA transfection reagent (Thermo Fisherr Scientific, Inc.), according to the manufacturer's instructions. The chondrocytes were serum-starved overnight followed by incubation at $37^{\circ} \mathrm{C}$ with SFN (50 $\mu \mathrm{M}$ ) for $24 \mathrm{~h}$. After $24 \mathrm{~h}$, the interference efficiency of the siRNA was evaluated by reverse transcription-quantitative PCR (RT-qPCR).

$R T-q P C R$. Total RNA was extracted from cells using TRIzol ${ }^{\circledR}$ reagent (Invitrogen; Thermo Fisherr Scientific, Inc.). The concentration and purity of RNA was evaluated by spectrometry at 260 and $280 \mathrm{~nm}$. The synthesis of cDNA was performed using the PrimeScript ${ }^{\mathrm{TM}}$ RT reagent kit [cat. no. 2312, Hangzhou Multisciences (Lianke) Biotech Co., Ltd.]. qPCR was performed using SYBR Green Real-Time PCR Master mix (Thermo Fisherr Scientific, Inc.) according to the manufacturer's protocol. The amplification conditions were as follows: SIRT1, $45 \mathrm{sec}$ at $94^{\circ} \mathrm{C}$ followed by 30 cycles of $30 \mathrm{sec}$ at $94^{\circ} \mathrm{C}, 30 \mathrm{sec}$ at $52^{\circ} \mathrm{C}$ and $60 \mathrm{sec}$ at $72^{\circ} \mathrm{C}$; GAPDH, $45 \mathrm{sec}$ at $94^{\circ} \mathrm{C}$ followed by 30 cycles of $30 \mathrm{sec}$ at $94^{\circ} \mathrm{C}, 30 \mathrm{sec}$ at $58^{\circ} \mathrm{C}$ and $60 \mathrm{sec}$ at $72^{\circ} \mathrm{C}$. Primers sequences, designed by Primer-Express V3.0 (Thermo Fisherr Scientific, Inc.), were as follows: SIRT1 forward, 5'-CTCTGAAAGTGAGACCAG TAGC-3' and reverse, 5'-TGTAGATGAGGCAAAGGTTCC-3' (product, $213 \mathrm{bp}$ ); and GAPDH forward, 5'-TCTTGCTCA GTGTCCTTGC-3' and reverse, 5'-CTTTGTCAAGCTCAT TTCCTGG-3' (product, $457 \mathrm{bp}$ ). Relative gene expression was analyzed using the $2^{-\Delta \Delta \mathrm{Cq}}$ method (36).

Immunofluorescence. Cells were rinsed using PBS and fixed in $4 \%$ paraformaldehyde for $15 \mathrm{~min}$ at $37^{\circ} \mathrm{C}$ after which they were washed three times using PBS. Then, they were treated with $0.1 \%$ Triton X-100 diluted in PBS for $15 \mathrm{~min}$ at room temperature. Next, chondrocytes were blocked using $10 \%$ goat serum (cat. no. SL038; Beijing Solarbio Science \& Technology Co., Ltd.; dissolved in PBS) for $4 \mathrm{~h}$ at $37^{\circ} \mathrm{C}$ and incubated at $4^{\circ} \mathrm{C}$ overnight in the presence of primary antibodies against collagen II (1:300; cat. no. 28459-1-AP; Wuhan Sanying Biotechnology). Following incubation, cells were exposed to Alexa Fluor ${ }^{\circledR}$ 594-labelled conjugated secondary antibodies (1:400; cat. no. IC1051T; R\&D Systems, Inc.) for $1.5 \mathrm{~h}$. Finally, they were exposed to DAPI $\left(10 \mu \mathrm{g} / \mathrm{ml} ; 37^{\circ} \mathrm{C}\right)$ (Beyotime Institute of Biotechnology) for $1 \mathrm{~min}$ and observed using a fluorescence microscope (magnification, x200; scale bar, $20 \mu \mathrm{m}$; Olympus Corporation). Fluorescence intensity was assessed using Image J software 6.0 (National Institutes of Health).

Immunohistochemical assay. The paraffin-embedded sections $(6 \mu \mathrm{m})$ were deparaffinized in xylene for $15 \mathrm{~min}$ at $37^{\circ} \mathrm{C}$ and rehydrated by incubation in series of graded ethanol $(100,95$, 85,75 and $0 \%$ ) for $5 \mathrm{~min}$ at $37^{\circ} \mathrm{C}$ and endogenous peroxidase. Then, sections were treated with $3 \%(\mathrm{v} / \mathrm{v})$ hydrogen peroxide for $10 \mathrm{~min}$ at $37^{\circ} \mathrm{C}$. Antigen retrieval of the sections was performed using 0.4\% pepsin (Sangon Biotech Co., Ltd.) in $5 \mathrm{mM} \mathrm{HCl}$ at $37^{\circ} \mathrm{C}$ for $20 \mathrm{~min}$. Then, sections were incubated in the presence of 10\% BSA (cat. no. ST025; Beyotime Institute of Biotechnology) for $45 \mathrm{~min}$ at room temperature, after which they were treated with primary antibodies against SIRT1 (1:500; cat. no. 2493; Cell Signaling Technology, Inc.) overnight at $4^{\circ} \mathrm{C}$. Finally, they were treated with horseradish peroxidase-conjugated secondary antibodies $(1: 1,000$; cat. no. SA00001-14; Wuhan Sanying Biotechnology) for $3 \mathrm{~h}$ at $4^{\circ} \mathrm{C}$. Following incubation at room temperature for $1 \mathrm{~h}$, the slices were rinsed $\left(5 \mathrm{~min}, 37^{\circ} \mathrm{C}\right)$, stained using a Metal Enhanced DAB Substrate kit $\left(2 \mathrm{~min}, 37^{\circ} \mathrm{C}\right.$; cat. no. DA1015; Beijing Solarbio Science \& Technology Co., Ltd.), dehydrated $\left(5 \mathrm{~min}, 37^{\circ} \mathrm{C}\right)$, mounted and examined under a microscope (magnification, $\mathrm{x} 40$; CX41; Olympus Corporation). Quantitative analysis was performed using Image-Pro Plus 6.0 software (Media Cybernetics, Inc.).

Animals. A total of 45 C57BL/6 male wild-type mice (age, 10 weeks; weight, $40 \mathrm{~g}$ ) were obtained from the Animal Center of the Chinese Academy of Sciences (Shanghai, China). The protocol for animal care and use conformed to The Guide for the Care and Use of Laboratory Animals of the National Institutes of Health published by the National Institutes of Health (NIH Publication No. 85-23, revised 1996) (37) and was approved by the Animal Care and Use Committee of Wenzhou Medical University. Experimental OA mice were established by destabilization of the medial meniscus (DMM) as previously described (38). Mice were housed under 12-h light/dark cycles and constant temperature $\left(21-23^{\circ} \mathrm{C}\right)$ and humidity (50-60\%). Water and food were provided ad libitum. Anesthesia was performed via intraperitoneal injection of $2 \%(\mathrm{w} / \mathrm{v})$ pentobarbital $(40 \mathrm{mg} / \mathrm{kg}$ ). Faint breathing, myasthenia, lack of independent reaction, cyanosis or coma were considered to indicate that mice were close to death; mice were euthanized by cervical dislocation. The joint capsule was carved followed by transection of the medial meniscotibial ligament of the right knee using microsurgical scissors. During surgery, the lateral meniscotibial ligament was always protected. As a control, the medial meniscotibial ligament was not transected while arthrotomy was performed in the left knee. Mice were randomly separated into three groups ( $n=15 /$ group): Control (sham-operated), DMM and DMM + SFN [mice were intraperitoneally administered with SFN at $20 \mathrm{mg} / \mathrm{kg}$ body weight $(39,40)]$. Parameters indicating the condition of mice were observed daily, including fur brightness, food and water intake, defecation and behavior. Furthermore, body weight was measured each week.

Histopathologic analysis. The extent of cartilage degeneration and synovitis in stained sections was assessed 


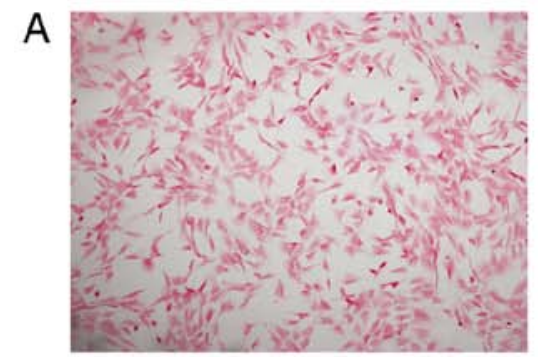

D

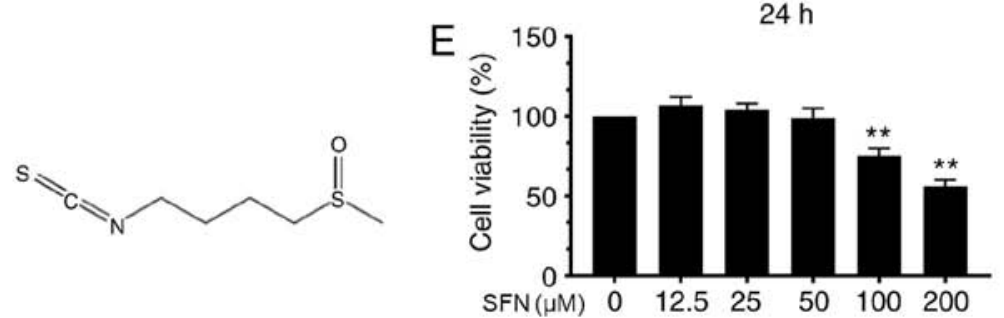

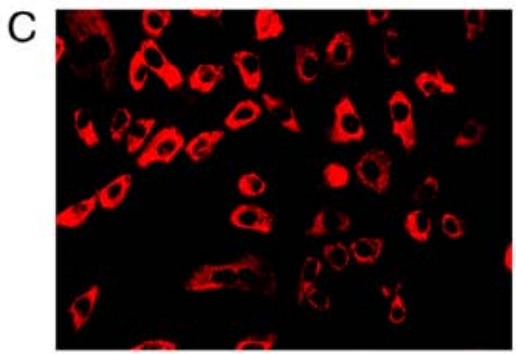

$48 \mathrm{~h}$

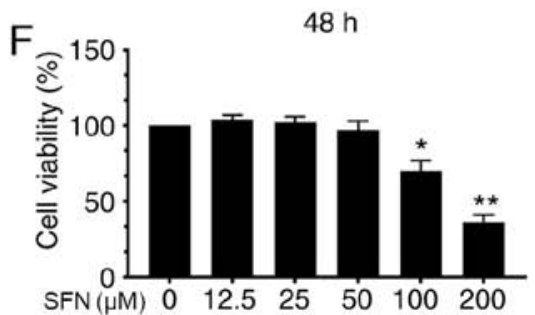

Figure 1. Effect of SFN on viability of chondrocytes. (A) Safranin O, (B) toluidine blue and (C) collagen II immunofluorescence (red) staining of mouse primary chondrocytes. (D) Chemical structure of SFN. Cell viability was determined via Cell Counting Kit- 8 assay at (E) 24 and (F) 48 h. Data are presented as the mean $\pm \mathrm{SD}(\mathrm{n}=3) .{ }^{*} \mathrm{P}<0.05,{ }^{* *} \mathrm{P}<0.01$ vs. $0 \mu \mathrm{M}$ SFN. SFN, sulforaphane.

using light microscopy with the Osteoarthritis Research Society International (OARSI) scoring system, as previously described (41).

Statistical analysis. The data are presented as mean $\pm \operatorname{SD}(n \geq 3)$. Statistical analysis was performed using GraphPad Prism version 5.0 software (GraphPad Software, Inc.). Comparisons between groups were performed using one-way ANOVA followed by Tukey's post hoc test. $\mathrm{P}<0.05$ was considered to indicate a statistically significant difference.

\section{Results}

Identification of mouse chondrocytes. Safranin O and toluidine blue staining were used to characterize mouse chondrocytes. Chondrocytes were stained red by Safranin O and the cytoplasm was stained purple by toluidine blue (Fig. 1A and B). In addition, collagen II in chondrocyte cytoplasm was stained red by immunofluorescence with no positive staining observed in the nucleus (Fig. 1C). These results imply that the cells isolated from mouse articular cartilage were chondrocytes.

Cytotoxicity of SFN on mouse chondrocytes. The chemical structure of SFN is shown in Fig. 1D. In order to determine the cytotoxic effects of SFN, CCK-8 assay was performed. Cells were incubated with increasing concentrations $(0.0,12.5$, $25.0,50.0,100.0$ and $200.0 \mu \mathrm{M}$ ) of SFN for 24 and $48 \mathrm{~h}$. SFN exhibited cytotoxic effects at $\geq 100 \mu \mathrm{M}$ after 24 or $48 \mathrm{~h}$ but not $<100 \mu \mathrm{M}$ (Fig. 1E and F). Therefore, $50 \mu \mathrm{M}$ was selected for use in subsequent experiments.

Effects of SFN on apoptosis in $\mathrm{H}_{2} \mathrm{O}_{2}$-treated chondrocytes. In order to determine the anti-apoptotic effect of SFN on $\mathrm{H}_{2} \mathrm{O}_{2}$-induced chondrocytes, the expression levels of Bax, Cleaved caspase- 3 and Bcl-2 were evaluated by western blot analysis. $\mathrm{H}_{2} \mathrm{O}_{2}$ elevated levels of Bax and Cleaved caspase-3, whereas Bcl-2 levels were downregulated (Fig. 2A-D). SFN downregulated levels of Bax and Cleaved caspase-3. Moreover, SFN significantly activated SIRT1 (Fig. 2A and E). These data indicate that SFN exerted an anti-apoptotic effect in mouse chondrocytes.

SFN alleviates ERS and apoptosis of $\mathrm{H}_{2} \mathrm{O}_{2}$-treated chondrocytes. In order to determine whether ERS in chondrocytes was associated with the cytoprotective effects of SFN on cartilage, cells were treated with $\mathrm{H}_{2} \mathrm{O}_{2}$, a typical ERS inducer. Expression levels of ERS-associated factors GRP78 and CHOP were measured. Western blotting assay exhibited increased expression levels of these proteins in response to $\mathrm{H}_{2} \mathrm{O}_{2}$; these effects were stopped by SFN (Fig. 3A-C). Moreover, apoptosis of cells in the $\mathrm{H}_{2} \mathrm{O}_{2}$ group was high compared with the control group. SFN treatment decreased cell apoptosis (Fig. 3D and E). Furthermore, $\mathrm{H}_{2} \mathrm{O}_{2}$ induced ROS production, which was inhibited by SFN (Fig. 3F).

SFN pretreatment enhances SIRT1 activity in $\mathrm{H}_{2} \mathrm{O}_{2}$-treated chondrocytes. In order to confirm the aforementioned results, SIRT1 siRNA was used to detect the potential mechanism of SFN on $\mathrm{H}_{2} \mathrm{O}_{2}$-stimulated chondrocytes. The mRNA expression levels of SIRT1 were significantly lower in SIRT1-silenced cells than in control cells (Fig. 4F). SIRT1 levels were elevated in the $\mathrm{H}_{2} \mathrm{O}_{2}+\mathrm{SFN}$ group but suppressed in the SIRT1 siRNA transfection group (Fig. 4A and E). Moreover, Bcl-2 levels were suppressed in the SIRT1 siRNA transfection group whereas the expression levels of Bax and Cleaved caspase-3 increased significantly (Fig. 4A-D). These results demonstrated the anti-apoptotic effect of SFN, which was mediated by activation of SIRT1.

SFN ameliorates OA development in a DMM mouse model. The chondroprotective effect of SFN on OA was investigated in vivo. A DMM mouse model was established, followed by intraperitoneal injection of $20 \mathrm{mg} / \mathrm{kg}$ SFN dissolved in DMSO every 2 days for 8 weeks. Safranin O staining was 

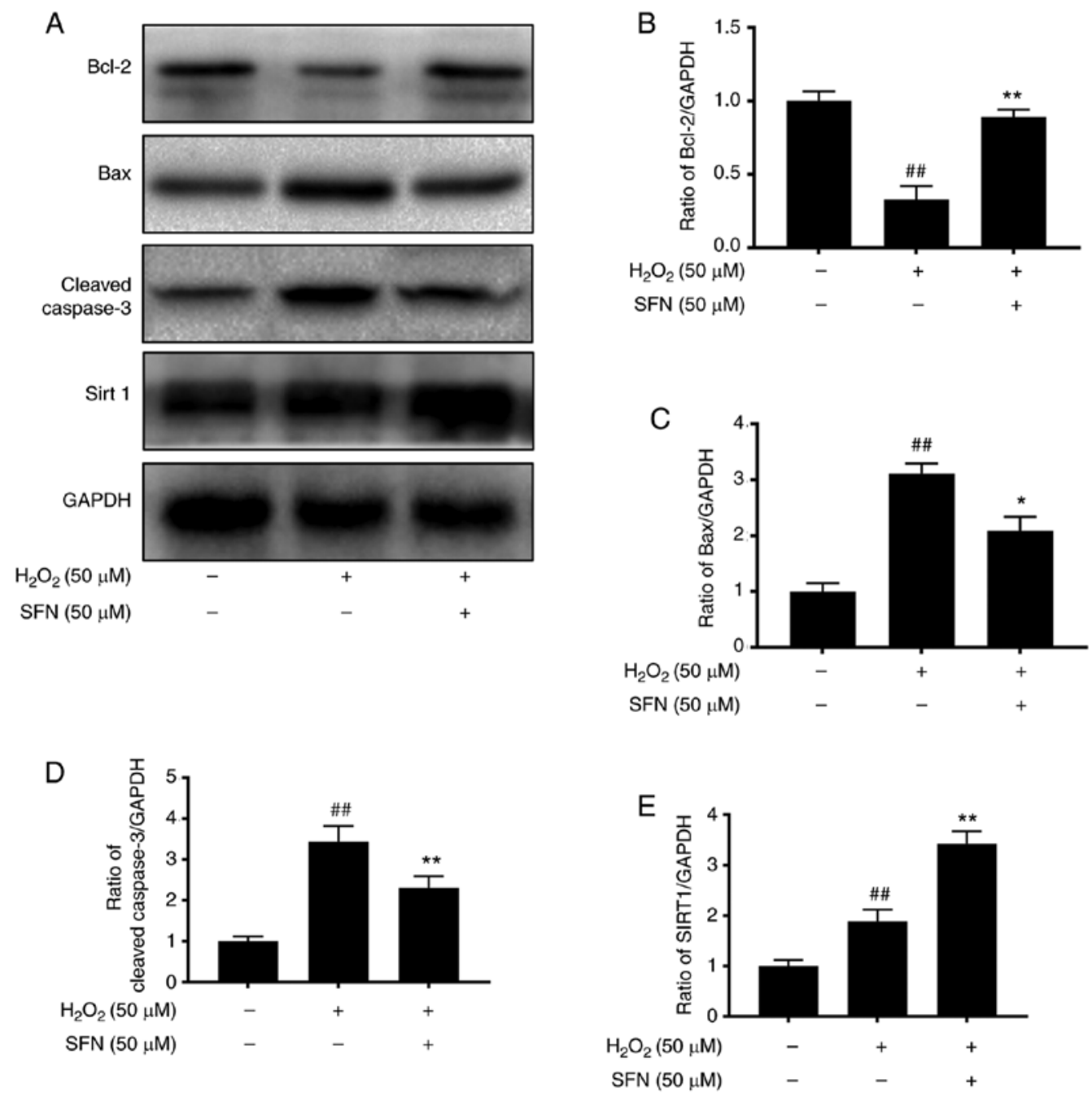

Figure 2. Effect of SFN on $\mathrm{H}_{2} \mathrm{O}_{2}$-induced apoptosis in mouse chondrocytes. (A) Western blot analysis assessed expression levels of (B) Bcl-2, (C) Bax, (D) Cleaved caspase-3 and (E) SIRT1. Data are presented as the mean $\pm \mathrm{SD}(\mathrm{n}=3)$. ${ }^{\# *} \mathrm{P}<0.01$ vs. control. ${ }^{*} \mathrm{P}<0.05,{ }^{* *} \mathrm{P}<0.01$ vs. $\mathrm{H}_{2} \mathrm{O}_{2}$-alone. SFN, sulforaphane; SIRT1, sirtuin 1 .

used to evaluate the effect of SFN on the development of OA. Compared with the sham group, the surface of the cartilage was notably destroyed in the DMM group (Fig. 5A). The degradation level of the cartilage matrix in the SFN group was lower than the OA but higher than the sham group. The OARSI scores in the SFN group were lower than those of the DMM group (Fig. 5B). Moreover, synovitis score in the DMM group was higher than in the sham group; SFN reversed this effect (Fig. 5A and C). In order to investigate the effect of SFN on SIRT1 expression in vivo, immunohistochemical staining for SIRT1 was performed (Fig. 5D and E). Compared with the DMM group, significantly more SIRT1-positive chondrocytes were detected in the DMM + SFN group. These results imply that SFN exhibited chondrocyte-protective effects during the progression of OA.

\section{Discussion}

OA is a chronic joint degradation disease that is characterized by long-term pain and joint limitation (42). Oral or topical non-steroidal anti-inflammatory drugs (such as ibuprofen and diclofenac sodium) are the primary therapeutic options for OA but only relieve clinical symptoms (3). These drugs are also associated with side effects, such as heart attack and stroke (43). Nonsteroidal anti-inflammatory drugs can only delay the progression of OA and surgery is the recommended option (44). Therefore, an agent that prevents OA progression, accompanied by fewer side effects, would be a potential therapeutic option for OA. Previous studies aimed to evaluate the efficacy of anti-apoptotic compounds, which exhibit few side effects $(45,46)$. Biochemical and biomechanical factors (such as ROS) induce chondrocyte apoptosis, as well as an imbalance between catabolism and anabolism of the ECM (47). Pharmacological administration of compounds (such as vitamin C and Glutathione) with the ability to regulate genetic mechanisms involved in apoptosis exhibit beneficial effects against OA development in vitro and in vivo (48). Therefore, the mechanism involved in chondrocyte apoptosis may be a potential therapeutic target for OA.

SFN, an isothiocyanate found in cruciferous vegetables, has been shown to exhibit anti-anxiety, sedative-hypnotic and anti-depression effects $(49,50)$. It is effective against oxidative-induced cardiomyocyte damage (51). Moreover, SFN has 


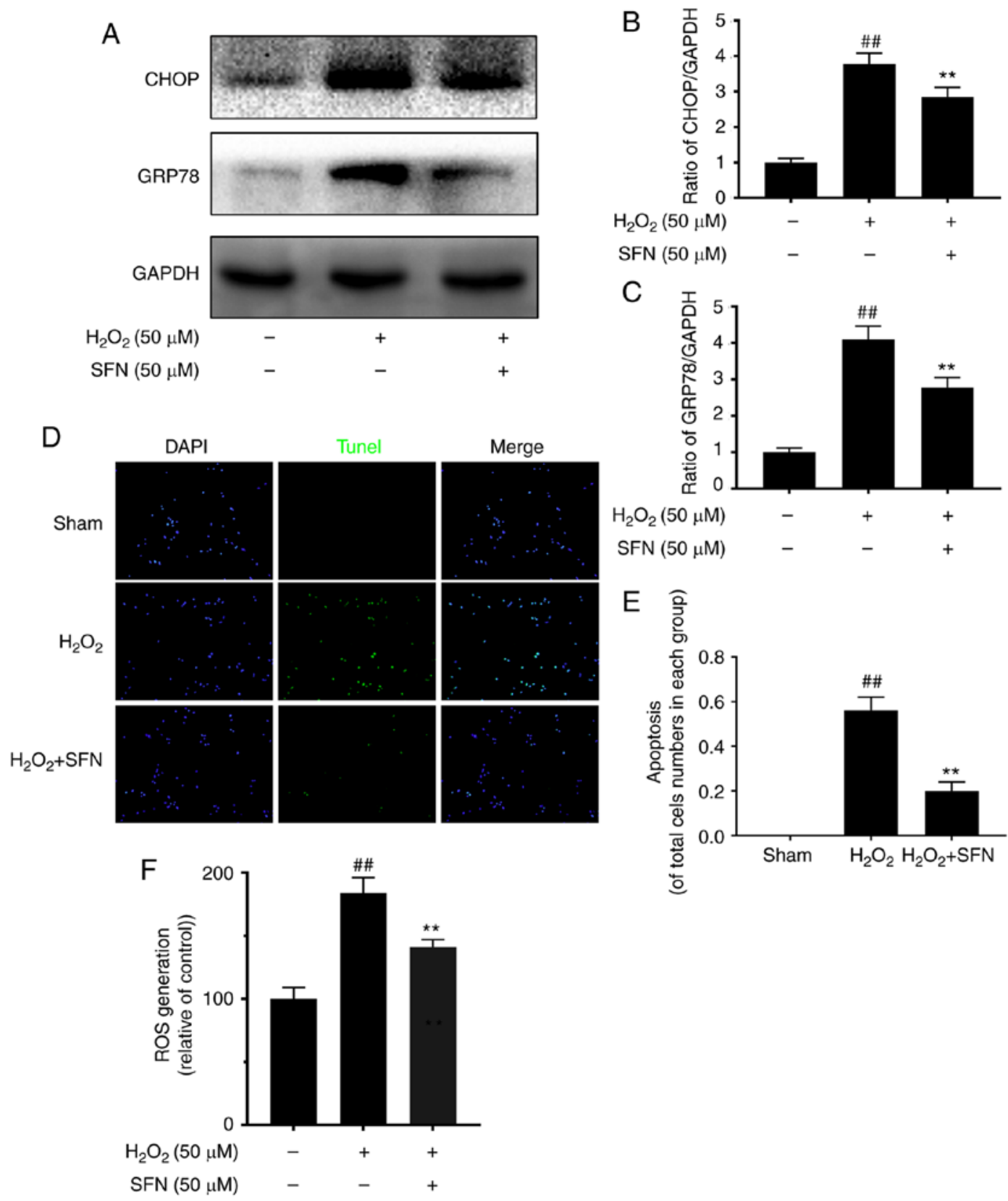

Figure 3. Effect of SFN on $\mathrm{H}_{2} \mathrm{O}_{2}$-induced endoplasmic reticulum stress and subsequent apoptosis in mouse chondrocytes. Chondrocytes were pretreated with $50 \mu \mathrm{M} \mathrm{SFN}$ for $24 \mathrm{~h}$ and incubated for $24 \mathrm{~h}$ in the presence or absence of $\mathrm{H}_{2} \mathrm{O}_{2}(50 \mu \mathrm{M})$. (A) Western blot analysis of levels of (B) CHOP and (C) GRP78 were assessed by western blotting. (D and E) Chondrocyte apoptosis was evaluated by In Situ Cell Death Detection kit (scale bar, $50 \mu \mathrm{m}$ ). (F) Quantification of ROS levels. Data are presented as the mean $\pm \mathrm{SD}(\mathrm{n}=3)$. ${ }^{\# /} \mathrm{P}<0.01$ vs. control. ${ }^{* *} \mathrm{P}<0.01$ vs. $\mathrm{H}_{2} \mathrm{O}_{2}$-alone. $\mathrm{SFN}$, sulforaphane; CHOP, C/EBP-homologous protein; GRP78, glucose-regulated protein 78; ROS, reactive oxygen species.

been shown to be effective against ERS in different models of cell injury, including chick yolk sac membrane and chorioallantoic membrane models (52). It inhibits ERS by suppressing the apoptosis of cells in type 1 diabetes mellitus (53). The anti-apoptotic effect of SFN was also found to be present in human hepatocytes (54). Wang et al (52) reported that SFN ameliorates ethanol-suppressed embryonic angiogenesis by alleviating excessive ROS production and ERS. Moreover, Li et al (26) documented that SFN suppresses expression levels of ERS-associated apoptosis proteins by activating SIRT1. The present study determined whether SFN ameliorates chondrocyte apoptosis and delays $\mathrm{OA}$ progression. $\mathrm{H}_{2} \mathrm{O}_{2}$, a key pro-apoptosis factor, was used to induce mitochondrial dysfunction, and upregulated the expression levels of caspase family proteins (55). The effect of SFN on ERS was evaluated in OA mouse chondrocytes. ER are found in the cytoplasm of all eukaryotic cells (56). ERS is involved in the development of OA (57). In the present study, the levels of ERS-associated apoptosis proteins (GRP78 and CHOP), Bax and Cleaved caspase-3 were elevated in chondrocytes following $\mathrm{H}_{2} \mathrm{O}_{2}$ treatment. However, pretreatment with SFN downregulated GRP78, CHOP, Bax and Cleaved caspase-3 levels and elevated Bcl-2 levels compared with the $\mathrm{H}_{2} \mathrm{O}_{2}$ group. These findings imply that SFN exerted chondroprotective effects against $\mathrm{H}_{2} \mathrm{O}_{2}$-induced apoptosis by decreasing ERS-dependent apoptosis. 


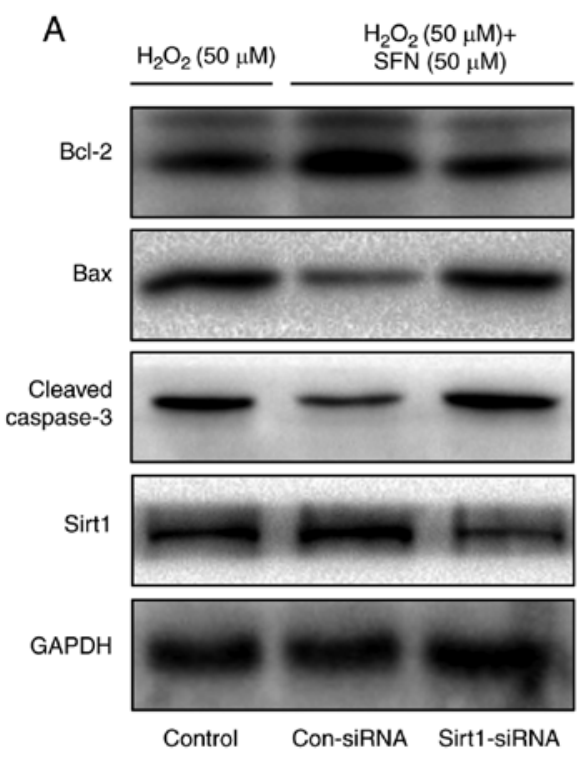

$\mathrm{D}$
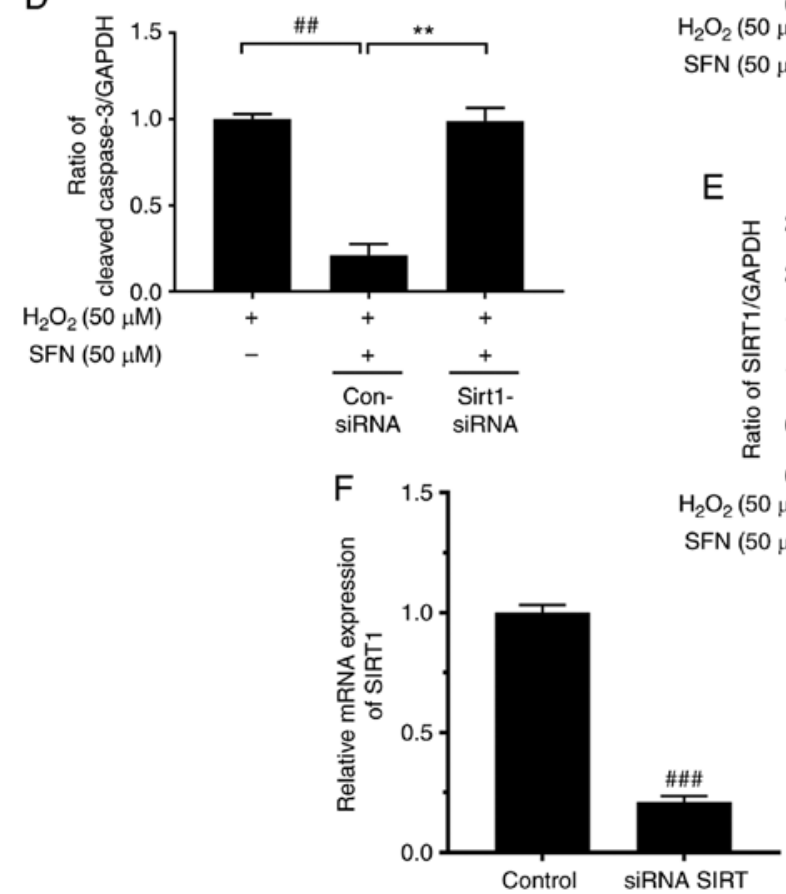

Figure 4. Protective effects of SFN in $\mathrm{H}_{2} \mathrm{O}_{2}$-induced chondrocytes are blocked by SIRT1-siRNA. (A) Following SIRT1 knockdown, western blot analysis was performed to detect expression levels of (B) Bcl-2, (C) Bax, (D) Cleaved caspase-3 and (E) SIRT1. (F) mRNA expression levels of SIRT1 were significantly inhibited in SIRT1-silenced cells. Data are presented as the mean $\pm \mathrm{SD}(\mathrm{n}=3) .{ }^{\# \#} \mathrm{P}<0.01,{ }^{\# \#} \mathrm{P}<0.001$ vs. Con; ${ }^{* * *} \mathrm{P}<0.01$ vs. $\mathrm{H}_{2} \mathrm{O}_{2}$ treatment. SFN, sulforaphane; SIRT1, sirtuin 1; si, small interfering; Con, control.

SIRT1 exerts anti-apoptotic effects in OA (58). Certain drugs, such as melatonin, curcumin and sildenafil, have been shown to exhibit chondroprotective effects in OA by modulating the SIRT1 signaling pathway $(59,60)$. The present study evaluated the effect of SIRT1 pathway signaling in SFN-induced chondroprotection. Compared with the $\mathrm{H}_{2} \mathrm{O}_{2}$ group, SIRT1 levels were significantly elevated in the SFN-pretreatment group.

SIRT1 siRNA was used to detect the potential mechanisms of SFN in $\mathrm{H}_{2} \mathrm{O}_{2}$-stimulated apoptotic chondrocytes. Consistent with previous studies, RNA interference against SIRT1 suppressed SFN-mediated inhibition of the anti-apoptosis effect $(61,62)$. Expression levels of SIRT1 were suppressed
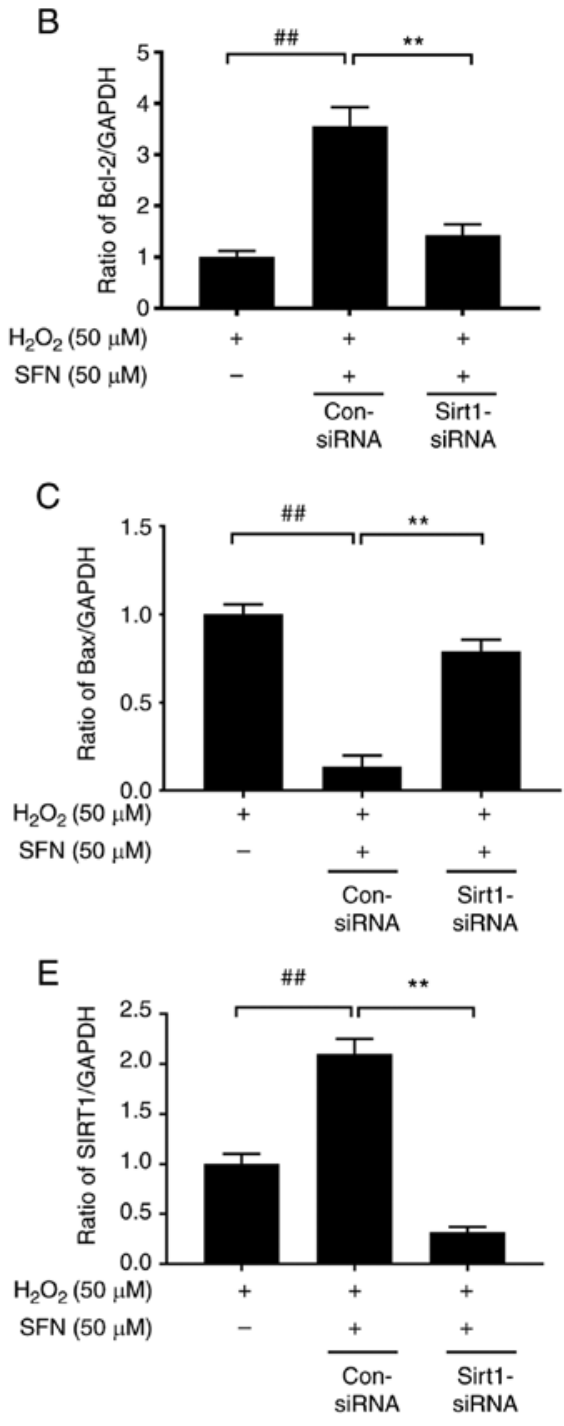

whereas levels of Cleaved caspase-3 were significantly elevated in the SIRT1 siRNA transfection group. These findings suggested that SIRT1 activation and ERS are associated with $\mathrm{H}_{2} \mathrm{O}_{2}$-induced apoptosis. A DMM mouse model was used to investigate the chondroprotective effects of SFN in vivo. Histological staining showed that SFN significantly alleviated disease progression in mice. This study has certain limitations; for example, flow cytometry analysis revealed the anti-apoptosis effect of SFN on OA, which needs to be further studied in future.

SFN protected chondrocytes against $\mathrm{H}_{2} \mathrm{O}_{2}$-induced apoptosis via the SIRT1 signaling pathway. Furthermore, SFN was shown to significantly relieve disease progression in a DMM 
A
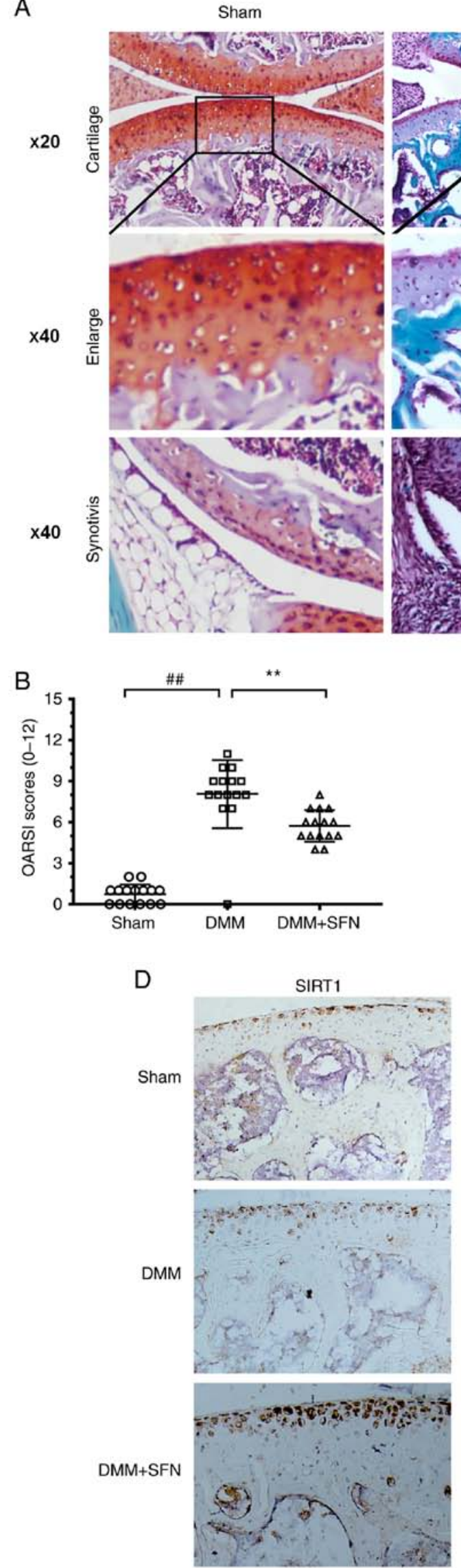

DMM
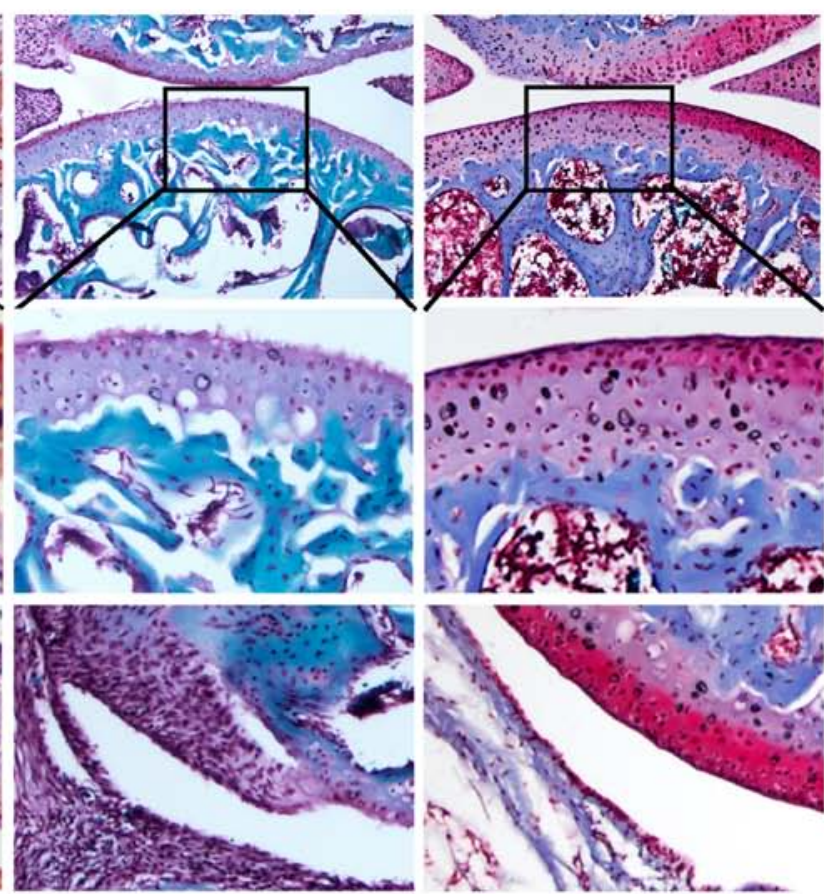

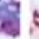
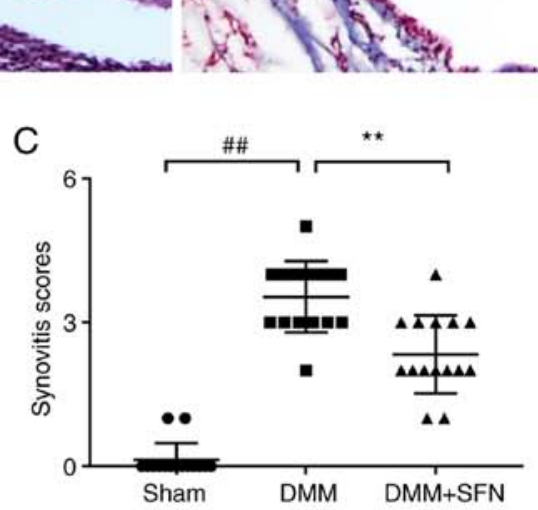

E

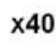

$\times 40$

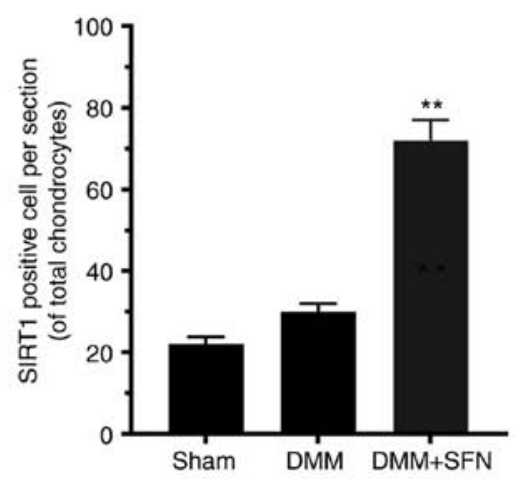

Figure 5. SFN inhibits OA development in a DMM mouse model. (A) Safranin O staining was used to evaluate histomorphometric differences between the sham, DMM and DMM + SFN groups. Magnification, x20 and x40. (B) OARSI and (C) synovitis scores for each cartilage sample. (D) Immunohistochemistry of SIRT1 in a DMM mouse model. (E) Quantification of SIRT1-positive cells in cartilage samples. Data are presented as the mean \pm SD $(n=15)$. ${ }^{\# \#} \mathrm{P}<0.01$ vs. sham, ${ }^{* *} \mathrm{P}<0.01$ vs. DMM group. SFN, sulforaphane; OA, osteoarthritis; DMM, destabilization of the medial meniscus; OARSI, Osteoarthritis Research Society International; SIRT1, sirtuin 1. 
mouse model. Therefore, SFN has a potential value in the prevention and treatment of OA.

\section{Acknowledgements}

Not applicable.

\section{Funding}

The present study was funded by the Basic Research on the Application of Suzhou Science and Technology Bureau (grant no. SYS201626), Key Technologies of People's Livelihood Technology of Suzhou Science and Technology Bureau (grant no. SS201764), Natural Science Foundation for Colleges and Universities in Jiangsu Province (grant no. 16KJB320009), Postgraduate Research \& Practice Innovation Program of Jiangsu Province (grant no. SJCX17_0658), The Second Affiliated Hospital of Soochow University Grant (grant no. XKQ2015003) and Orthopedics Department and Postgraduate Research \& Practice Innovation Program of Jiangsu Province (grant no. KYCX18_ 2530).

\section{Availability of data and materials}

The datasets used and/or analyzed during the current study are available from the corresponding author on reasonable request.

\section{Authors' contributions}

MC and LH wrote the manuscript. YL and LL designed and supervised the study. MC, LH, YL and QD performed the experiments. MC, YL and LL analyzed and interpreted the experimental data. All the authors discussed the results and commented on the manuscript. MC LH, YL and QD confirm the authenticity of all the raw data. All authors read and approved the final manuscript.

\section{Ethics approval and consent to participate}

The protocol for animal care and use conformed to The Guide for the Care and Use of Laboratory Animals of the National Institutes of Health and was approved by the Animal Care and Use Committee of Wenzhou Medical University (ethics approval no. wydw2019-0377).

\section{Patient consent for publication}

Not applicable.

\section{Competing interests}

The authors declare that they have no competing interests.

\section{References}

1. Xing D, Xu Y, Liu Q, Ke Y, Wang B, Li Z and Lin J: Osteoarthritis and all-cause mortality in worldwide populations: Grading the evidence from a meta-analysis. Sci Rep 6: 24393, 2016.

2. Hunter DJ and Felson DT: Osteoarthritis. BMJ 332: 639-642, 2006.
3. Lane NE, Shidara K and Wise BL: Osteoarthritis year in review 2016: Clinical. Osteoarthritis Cartilage 25: 209-215, 2017.

4. Reginster JY: The prevalence and burden of arthritis. Rheumatology (Oxford) 41 (Suppl 1): S3-S6, 2002.

5. Mobasheri A: The future of osteoarthritis therapeutics: Emerging biological therapy. Curr Rheumatol Rep 15: 385, 2013.

6. Portal-Núñez S, Esbrit P, Alcaraz MJ and Largo R: Oxidative stress, autophagy, epigenetic changes and regulation by miRNAs as potential therapeutic targets in osteoarthritis. Biochem Pharmacol 108: 1-10, 2016.

7. Xia H, Cao D, Yang F, Yang W, Li W, Liu P, Wang S and Yang F: Jiawei Yanghe decoction ameliorates cartilage degradation in vitro and vivo via $\mathrm{Wnt} / \beta$-catenin signaling pathway. Biomed Pharmacother 122: 109708, 2020.

8. Dai M, Sui B, Xue Y, Liu X and Sun J: Cartilage repair in degenerative osteoarthritis mediated by squid type II collagen via immunomodulating activation of M2 macrophages, inhibiting apoptosis and hypertrophy of chondrocytes. Biomaterials 180: 91-103, 2018.

9. Lee AS, Ellman MB, Yan D, Kroin JS, Cole BJ, van Wijnen AJ and Im HJ: A current review of molecular mechanisms regarding osteoarthritis and pain. Gene 527: 440-447, 2013.

10. Musumeci G, Castrogiovanni P, Trovato FM, Weinberg AM, Al-Wasiyah MK, Alqahtani MH and Mobasheri A: Biomarkers of chondrocyte apoptosis and autophagy in osteoarthritis. Int J Mol Sci 16: 20560-20575, 2015.

11. Bondeson J, Wainwright S, Hughes $\mathrm{C}$ and Caterson B: The regulation of the ADAMTS4 and ADAMTS5 aggrecanases in osteoarthritis: A review. Clin Exp Rheumatol 26: 139-145, 2008.

12. Sharif M, Whitehouse A, Sharman P, Perry M and Adams M: Increased apoptosis in human osteoarthritic cartilage corresponds to reduced cell density and expression of caspase- 3 . Arthritis Rheum 50: 507-515, 2004.

13. Claudio N, Dalet A, Gatti E and Pierre P: Mapping the crossroads of immune activation and cellular stress response pathways. EMBO J 32: 1214-1224, 2013.

14. Feng K, Chen Z, Pengcheng L, Zhang S and Wang X: Quercetin attenuates oxidative stress-induced apoptosis via SIRT1/AMPK-mediated inhibition of ER stress in rat chondrocytes and prevents the progression of osteoarthritis in a rat model. J Cell Physiol 234: 18192-18205, 2019.

15. Bernales S, Papa FR and Walter P: Intracellular signaling by the unfolded protein response. Annu Rev Cell Dev Biol 22: 487-508, 2006.

16. Wictome M, Henderson I, Lee AG and East JM: Mechanism of inhibition of the calcium pump of sarcoplasmic reticulum by thapsigargin. Biochem J 283: 525-529, 1992.

17. Hetz C: The unfolded protein response: Controlling cell fate decisions under ER stress and beyond. Nat Rev Mol Cell Biol 13: 89-102, 2012.

18. Huang L, Xie $\mathrm{H}$ and Liu H: Endoplasmic reticulum stress, diabetes mellitus, and tissue injury. Curr Protein Pept Sci 15: 812-818, 2014

19. Chen D, Wang Y and Chin ER: Activation of the endoplasmic reticulum stress response in skeletal muscle of G93A*SOD1 amyotrophic lateral sclerosis mice. Front Cell Neurosci 9: 170, 2015.

20. Rasmussen ML, Kline LA, Park KP, Ortolano NA, Romero-Morales AI, Anthony CC, Beckermann KE and Gama V: A non-apoptotic function of MCL-1 in promoting pluripotency and modulating mitochondrial dynamics in stem cells. Stem Cell Reports 10: 684-692, 2018.

21. Zhao CQ, Zhang YH, Jiang SD, Jiang LS and Dai LY: Both endoplasmic reticulum and mitochondria are involved in disc cell apoptosis and intervertebral disc degeneration in rats. Age (Dordr) 32: 161-177, 2010.

22. Lin J, Zhuge J, Zheng X, Wu Y, Zhang Z, Xu T, Meftah Z, Xu H, Wu Y, Tian N, et al: Urolithin A-induced mitophagy suppresses apoptosis and attenuates intervertebral disc degeneration via the AMPK signaling pathway. Free Radic Biol Med 150: 109-119, 2020.

23. Shah SA, Khan M, Jo MH, Jo MG, Amin FU and Kim MO: Melatonin stimulates the SIRT1/Nrf2 signaling pathway counteracting lipopolysaccharide (LPS)-induced oxidative stress to rescue postnatal rat brain. CNS Neurosci Ther 23: 33-44, 2017.

24. Morris BJ: Seven sirtuins for seven deadly diseases of aging. Free Radic Biol Med 56: 133-171, 2013.

25. Rajendran R, Garva R, Krstic-Demonacos M and Demonacos C: Sirtuins: Molecular traffic lights in the crossroad of oxidative stress, chromatin remodeling, and transcription. J Biomed Biotechnol 2011: 368276, 2011. 
26. Li YP, Wang SL, Liu B, Tang L, Kuang RR, Wang XB, Zhao C, Song XD, Cao XM, Wu X, et al: Sulforaphane prevents rat cardiomyocytes from hypoxia/reoxygenation injury in vitro via activating SIRT1 and subsequently inhibiting ER stress. Acta Pharmacol Sin 37: 344-353, 2016.

27. Katto J, Engel N, Abbas W, Herbein G and Mahlknecht U: Transcription factor NFKB regulates the expression of the histone deacetylase SIRT1. Clin Epigenetics 5: 11, 2013.

28. Feng K, Ge Y, Chen Z, Li X, Liu Z, Li X, Li H, Tang T, Yang F and Wang X: Curcumin inhibits the PERK-eIF2 $\alpha$-CHOP pathway through promoting SIRT1 expression in oxidative stress-induced rat chondrocytes and ameliorates osteoarthritis progression in a rat model. Oxid Med Cell Longev 2019: 8574386, 2019.

29. Danilov CA, Chandrasekaran K, Racz J, Soane L, Zielke C and Fiskum G: Sulforaphane protects astrocytes against oxidative stress and delayed death caused by oxygen and glucose deprivation. Glia 57: 645-656, 2009.

30. Nguyen B, Luong L, Naase H, Vives M, Jakaj G, Finch J, Boyle J, Mulholland JW, Kwak JH, Pyo S, et al: Sulforaphane pretreatment prevents systemic inflammation and renal injury in response to cardiopulmonary bypass. J Thorac Cardiovase Surg 148: 690-697, 2014.

31. Forster T, Rausch V, Zhang Y, Isayev O, Heilmann K, Schoensiegel F, Liu L, Nessling M, Richter K, Labsch S, et al: Sulforaphane counteracts aggressiveness of pancreatic cancer driven by dysregulated $\mathrm{Cx} 43$-mediated gap junctional intercellular communication. Oncotarget 5: 1621-1634, 2014.

32. Ho JN, Yoon HG, Park CS, Kim S, Jun W, Choue R and Lee J: Isothiocyanates ameliorate the symptom of heart dysfunction and mortality in a murine AIDS model by inhibiting apoptosis in the left ventricle. J Med Food 15: 781-787, 2012.

33. Davidson RK, Jupp O, de Ferrars R, Kay CD, Culley KL, Norton R, Driscoll C, Vincent TL, Donell ST, Bao Y and Clark IM: Sulforaphane represses matrix-degrading proteases and protects cartilage from destruction in vitro and in vivo. Arthritis Rheum 65: 3130-3140, 2013.

34. Davidson RK, Green J, Gardner S, Bao Y, Cassidy A and Clark IM: Identifying chondroprotective diet-derived bioactives and investigating their synergism. Sci Rep 8: 17173, 2018.

35. Zheng G, Zhan Y, Tang Q, Chen T, Zheng F, Wang H, Wang J, Wu D, Li X, Zhou Y, et al: Monascin inhibits IL-1 $\beta$ induced catabolism in mouse chondrocytes and ameliorates murine osteoarthritis. Food Funct 9: 1454-1464, 2018.

36. Livak KJ and Schmittgen TD: Analysis of relative gene expression data using real-time quantitative PCR and the 2(-Delta Delta C(T)) method. Methods 25: 402-408, 2001.

37. The Guide for the Care and Use of Laboratory Animals of the National Institutes of Health published by the National Institutes of Health. NIH Publication No. 85-23, revised 1996

38. Glasson SS, Blanchet TJ and Morris EA: The surgical destabilization of the medial meniscus (DMM) model of osteoarthritis in the 129/SvEv mouse. Osteoarthritis Cartilage 15: 1061-1069, 2007.

39. Yoo IH, Kim MJ, Kim J, Sung JJ, Park ST and Ahn SW: The anti-inflammatory effect of sulforaphane in mice with experimental autoimmune encephalomyelitis. J Korean Med Sci 34 e197, 2019

40. Huo L, Su Y, Xu G, Zhai L and Zhao J: Sulforaphane protects the male reproductive system of mice from obesity-induced damage: Involvement of oxidative stress and autophagy. Int J Environ Res Public Health 16: 3759, 2019.

41. Glasson SS, Chambers MG, Van Den Berg WB and Little CB: The OARSI histopathology initiative-recommendations for histological assessments of osteoarthritis in the mouse. Osteoarthritis Cartilage 18 (Suppl 3): S17-S23, 2010

42. French HP, Galvin R, Horgan NF and Kenny RA: Prevalence and burden of osteoarthritis amongst older people in Ireland: Findings from The Irish LongituDinal Study on Ageing (TILDA). Eur J Public Health 26: 192-198, 2016.

43. Glyn-Jones S, Palmer AJ, Agricola R, Price AJ, Vincent TL, Weinans H and Carr AJ: Osteoarthritis. Lancet 386: 376-387, 2015.

44. Johnson VL and Hunter DJ: The epidemiology of osteoarthritis. Best Pract Res Clin Rheumatol 28: 5-15, 2014
45. Asada S, Fukuda K, Nishisaka F, Matsukawa M and Hamanisi C: Hydrogen peroxide induces apoptosis of chondrocytes; involvement of calcium ion and extracellular signal-regulated protein kinase. Inflamm Res 50: 19-23, 2001.

46. Kim EN, Lee HS and Jeong GS: Cudratricusxanthone O Inhibits $\mathrm{H}_{2} \mathrm{O}_{2}$-Induced cell damage by activating Nrf2/HO-1 pathway in human chondrocytes. Antioxidants (Basel) 9: 788, 2020.

47. Sutipornpalangkul W, Morales NP and Harnroongroj T: Free radicals in primary knee osteoarthritis. J Med Assoc Thai 92 (Suppl 6): S268-S274, 2009.

48. Hadjigogos K: The role of free radicals in the pathogenesis of rheumatoid arthritis. Panminerva Med 45: 7-13, 2003.

49. Russo M, Spagnuolo C, Russo GL, Skalicka-Woźniak K, Daglia M, Sobarzo-Sánchez E, Nabavi SF and Nabavi SM: Nrf2 targeting by sulforaphane: A potential therapy for cancer treatment. Crit Rev Food Sci Nutr 58: 1391-1405, 2018.

50. Sita G, Hrelia P, Graziosi A and Morroni F: Sulforaphane from cruciferous vegetables: Recent advances to improve glioblastoma treatment. Nutrients 10: 1755, 2018.

51. Corssac GB, Campos-Carraro C, Hickmann A, da Rosa Araujo AS, Fernandes RO and Belló-Klein A: Sulforaphane effects on oxidative stress parameters in culture of adult cardiomyocytes. Biomed Pharmacother 104: 165-171, 2018.

52. Wang G, Nie JH, Bao Y and Yang X: Sulforaphane rescues ethanol-suppressed angiogenesis through oxidative and endoplasmic reticulum stress in chick embryos. J Agric Food Chem 66: 9522-9533, 2018.

53. Pu D, Zhao Y, Chen J, Sun Y, Lv A, Zhu S, Luo C, Zhao K and Xiao Q: Protective effects of sulforaphane on cognitive impairments and AD-like lesions in diabetic mice are associated with the upregulation of Nrf2 transcription activity. Neuroscience 381: 35-45, 2018.

54. Tubbs E, Axelsson AS, Vial G, Wollheim CB, Rieusset J and Rosengren AH: Sulforaphane improves disrupted ER-mitochondria interactions and suppresses exaggerated hepatic glucose production. Mol Cell Endocrinol 461: 205-214, 2018.

55. Chen Z, Yuan Q, Xu G, Chen H, Lei H and Su J: Effects of quercetin on proliferation and $\mathrm{H}_{2} \mathrm{O}_{2}$-induced apoptosis of intestinal porcine enterocyte cells. Molecules 23: 2012, 2018.

56. Chandrika BB, Yang C, Ou Y, Feng X, Muhoza D, Holmes AF, Theus S, Deshmukh S, Haun RS and Kaushal GP: Endoplasmic reticulum stress-induced autophagy provides cytoprotection from chemical hypoxia and oxidant injury and ameliorates renal ischemia-reperfusion injury. PLoS One 10: e0140025, 2015.

57. Tang Q, Zheng G, Feng Z, Chen Y, Lou Y, Wang C, Zhang X, Zhang Y, Xu H, Shang P and Liu H: Trehalose ameliorates oxidative stress-mediated mitochondrial dysfunction and ER stress via selective autophagy stimulation and autophagic flux restoration in osteoarthritis development. Cell Death Dis 8: e3081, 2017.

58. Deng Z, Li Y, Liu H, Xiao S, Li L, Tian J, Cheng C, Zhang G and Zhang F: The role of sirtuin 1 and its activator, resveratrol in osteoarthritis. Biosci Rep 39: BSR20190189, 2019.

59. Guo JY, Li F, Wen YB, Cui HX, Guo ML, Zhang L, Zhang YF, Guo YJ and Guo YX: Melatonin inhibits Sirt1-dependent NAMPT and NFAT5 signaling in chondrocytes to attenuate osteoarthritis. Oncotarget 8: 55967-55983, 2017.

60. Hu A, Liu HB, Mlynski R, Plontke S, Zhang JF, Dai WJ, Duan JL, Fan JP, Zheng HL, Xu WH, et al: Therapeutic ultrasound potentiates the anti-nociceptive and anti-inflammatory effects of curcumin to postoperative pain via Sirt1/NF- $\kappa \mathrm{B}$ signaling pathway. Am J Transl Res 10: 3099-3110. eCollection, 2018.

61. Li T, Pang Q, Liu Y, Bai M, Peng Y and Zhang Z: Sulforaphane protects human umbilical vein endothelial cells from oxidative stress via the miR-34a/SIRT1 axis by upregulating nuclear factor erythroid-2-related factor 2. Exp Ther Med 21: 186, 2021.

62. Sun X, Mi L, Liu J, Song L, Chung LF and Gan N: Sulforaphane prevents microcystin-LR-induced oxidative damage and apoptosis in BALB/c mice. Toxicol Appl Pharmacol 255: 9-17, 2011.

This work is licensed under a Creative Commons Attribution-NonCommercial-NoDerivatives 4.0 International (CC BY-NC-ND 4.0) License. 